\title{
Classifying interactions in a synthetic bacterial community is hindered by inhibitory growth medium
}

\author{
Andrea R. Dos Santos ${ }^{1}$, Rita Di Martino ${ }^{1}$, Samuele Testa ${ }^{1}$, and Sara Mitri ${ }^{1,2, \mathbb{}}$ \\ ${ }^{1}$ Department of Fundamental Microbiology, University of Lausanne, Switzerland \\ ${ }^{2}$ Swiss Institute for Bioinformatics
}

\begin{abstract}
Predicting the fate of a microbial community and its 38 member species relies on understanding the nature of their in- 39 teractions. However, designing simple assays that distinguish between interaction types can be challenging. Here, we performed spent media assays based on the predictions of a mathematical model to decipher the interactions between four bacterial species: Agrobacterium tumefaciens (At), Comamonas testosteroni $(C t)$, Microbacterium saperdae (Ms) and Ochrobactrum anthropi $(\mathrm{Oa})$. While most experimental results matched model predictions, the behavior of $\boldsymbol{C t}$ did not: its lag phase was reduced ${ }^{46}$ in the pure spent media of $\boldsymbol{A t}$ and $\mathbf{M s}$, but prolonged again when ${ }^{47}$ we replenished with our growth medium. Further experiments 48 showed that the growth medium actually delayed the growth 49 of $\boldsymbol{C t}$, leading us to suspect that $\boldsymbol{A t}$ and $\boldsymbol{M s}$ could alleviate this ${ }_{50}$ inhibitory effect. There was, however, no evidence supporting such "cross-detoxification" and instead, we identified metabolites secreted by $A t$ and $M s$ that were then consumed or "crossfed" by $C t$, shortening its lag phase. Our results highlight that even simple, defined growth media can have inhibitory effects on some species and that such negative effects need to be included 55 in our models. Based on this, we present new guidelines to cor- 56 rectly distinguish between different interaction types, such as 57 cross-detoxification and cross-feeding.
\end{abstract}

Cross-feeding | Detoxification | Consumer-resource model | Spent media | Ex- 59 perimental design

Correspondence: sara.mitri@unil.ch

\section{Introduction}

their traits (their evolution) (1-4). Positive interactions occur when one species improves the environment for another, either by reducing its adverse effects or by producing compounds that enhance the other's growth (1). Whether these improvements also benefit the acting species and/or are costly can affect evolutionary dynamics. For example, siderophores or nutrient-degrading enzymes are useful to their producers as well as other species, but are quite costly (5). Nonproducing mutants can then invade the population of producers and destabilize the interaction. But positive interactions can remain stable over time if they are not exploitable: a species may take up nutrients that alter the $\mathrm{pH}$ to another species' benefit $(6,7)$, or secrete cost-less metabolic byproducts, which can be cross-fed by other co-inhabiting species $(8,9)$.

Predicting the long-term fate of competitive interactions is equally mechanism-dependent. Competition can be due to one species enhancing harmful conditions (e.g. production of bacteriocins), or removing beneficial ones (e.g. competition for nutrients) $(1,10)$. Under the latter, known as "exploitative competition", species compete for resources, and we expect them to evolve to occupy separate niches and compete less (11-14). Under more aggressive "interference competition", the production of toxins, antibiotics or phage-like particles may result in arms races and species extinctions $(10,15)$. Competitive interactions often rely on direct cell-to-cell contact (15). In sum, even if positive and competitive interactions are easily measurable at the population level, understanding the mechanisms underlying these measured effects can change the predictions of long-term dynamics or environmental changes.

In natural communities, interactions occur simultaneously between many species, with little evidence of which molecule was produced or consumed by which species, and which species it affects in which way. Identifying interactions and their molecular mechanisms in such complex webs 
is clearly quite challenging, but can possibly be achieved in 122 a high-throughput manner using spent media (SM) assays, 123 which we will show can distinguish between interaction types 124 without needing to distinguish interacting species, e.g. by fluorescently labelling them. To develop and test the utility of 125 these assays, small synthetic microbial ecosystems of up to a few dozen species are more practical (16-20): Inter-species interactions are easier to disentangle and control, especially since the chemistry of the environment can be designed, and community members can be genetically engineered or selected to exhibit specific interactions (21-26). Their simplicity also allows for parameter estimation in mathematical models to predict community dynamics $(22,27-30)$.

Here, we aimed to decipher the interactions in a synthetic community we studied previously, composed of four ${ }_{135}$ bacterial species: Agrobacterium tumefaciens (At), Coma- ${ }_{136}$ monas testosteroni $(\mathrm{Ct})$, Microbacterium saperdae $(\mathrm{Ms})$, and ${ }_{137}$ Ochrobactrum anthropi $(\mathrm{Oa})(31)$. This community was ${ }_{138}$ dominated by positive interactions when grown in a chem- ${ }_{139}$ ically complex environment. Here, we sought to provide ${ }_{140}$ a more controlled environment and used a defined minimal ${ }_{141}$ medium (MM) to study the mechanisms behind the interactions between the four species. As we were initially inter- ${ }_{143}$ ested in chemical and metabolic interactions that do not re- ${ }_{144}$ quire cell-to-cell contact, we grew each species in the $\mathrm{SM}_{145}$ of all others using an experimental design that allows us to ${ }_{146}$ distinguish between interaction types (e.g. exploitative vs. ${ }_{147}$ interference competition). Ideally, these simple assays would suffice to identify all types of pairwise interactions, without the need for detailed chemical analyses of secreted and con- ${ }_{150}$ sumed molecules.

We found two strong positive interactions mirroring our pre- 152 vious work: the pure SM of $A t$ and $M s$ shortened the lag ${ }_{153}$ phase of $C t$. However, we were surprised that this positive 154 effect was lost and even reversed if the spent media were re- 155 plenished with the original growth medium (MM). Further ${ }_{156}$ investigation revealed that the no-carbon (NC) compounds 157 (that cannot be used as carbon sources) in the replenished ${ }_{158}$ SM delay C $t$ 's growth despite the presence of enough avail- ${ }_{159}$ able carbon sources and the SM. We then wondered whether 160 At and $M s$ might remove the inhibitory compounds from the ${ }_{161}$ environment for $C t$, allowing it to grow sooner. Although ${ }_{162}$ such cross-detoxification seemed like the most parsimonious ${ }_{163}$ explanation, we found no evidence to support it. Instead, ${ }_{164}$ using untargeted liquid chromatography-mass spectrometry 165 (LC-MS), we identified at least three molecules secreted by 166 $A t$ and/or $M s$ that could be metabolised by $C t$ and shortened ${ }_{167}$ its lag phase. Our findings show that pinpointing the nature 168 of positive interactions can be quite challenging, as different effects can be intimately intertwined: cross-feeding could alleviate the negative effects of a challenging environment.

\section{Results}

\section{At and $\mathrm{Oa}$ responded to others following anticipated} scenarios. To identify the interactions between the four species in our simplified medium, we conducted spent media (SM) assays by growing each species alone until stationary phase in a minimal medium (MM) containing a no-carbon part $(\mathrm{NC}=$ salts and trace metals) and two carbon sources (CS): glucose and citric acid (MM = NC + CS, see Methods). We then removed the cells by filtration and grew each species in all other resulting spent media (SM). We compared the growth in SM (condition IV) to the growth in a medium comprising only the NC compounds (condition I), a positive control of MM (condition II) and two other conditions (Fig. 1A, left): First (condition III), we diluted (1:1) the SM in $2 \times$ NC so that the species have access to at least the same concentrations of salts and trace metals as in the fresh MM, and second (condition V), SM was diluted (1:1) in $2 \times \mathrm{MM}$ to contain at least the original concentration of carbon sources, salts and trace metals.

Our expectations for these five different experimental conditions were established using a mathematical model where we simulated the outcome of six interaction types: exploitative competition, exploitative with interference competition, exploitative competition with cross-feeding, niche separation, niche separation with interference competition, and niche separation with cross-feeding (Figs. S1, S2). We calculated the area under each simulated growth curve, and compared it to the negative and positive controls (I and II) (Fig. 1A). For example, if two species compete for the same carbon source or a species is grown in its own SM (exploitative competition, EC), the carbon source in the model is depleted such that condition III and IV are similar to I. The replenishment of the carbon sources in condition $\mathrm{V}$ restores growth to the level of the positive control II. The remaining base expectations are shown in Figs. 1A and S2.

The spent media experiments were analyzed by calculating the area under the $\mathrm{OD}_{600}$ growth curves. At and $\mathrm{Oa}$ 's growth could be classified according to the six anticipated scenarios (Fig. 1B, S3, S4). We have omitted data on $M s$, as quantifying its growth was problematic due to contradictions between our measurements (Fig. S3, S5). Surprisingly, the growth patterns of $C t$ did not correspond to any of the expected scenarios (Fig. 1B).

We first verified the behavior of $A t$ and $O a$ by quantifying 

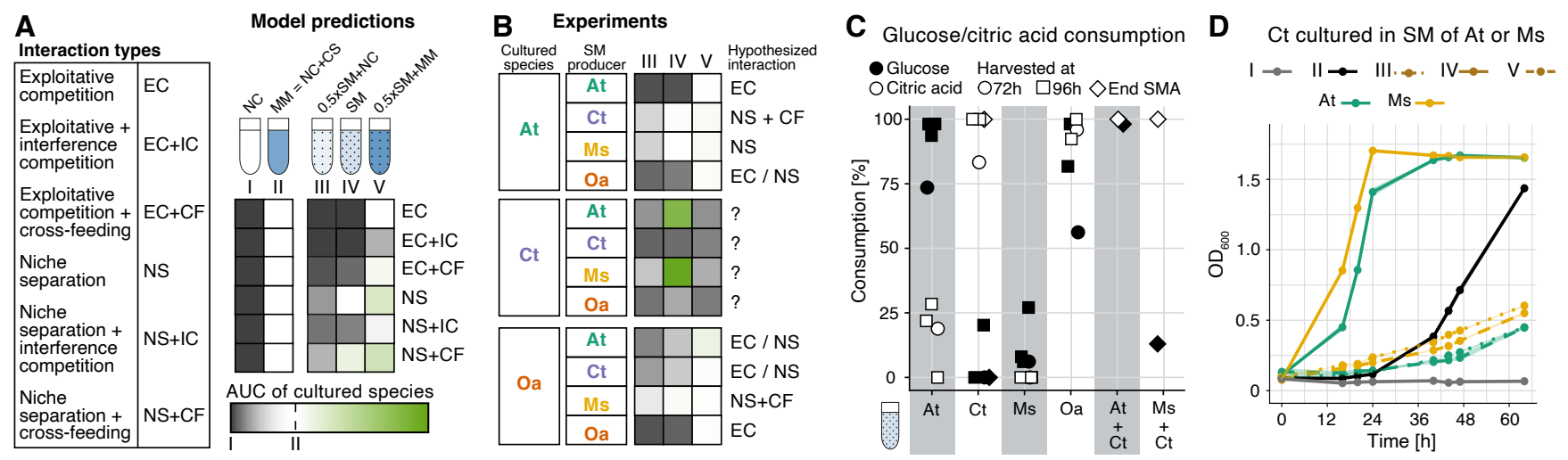

Fig. 1. Spent media assays. (A) We used our model to simulate the growth of species under different types of positive or negative interactions, as listed in the table and illustrated in Fig. S1. Five simulated growth conditions allowed us to distinguish between these four different interaction types: I. no-carbon (NC) medium containing only salts and trace metals, but no carbon sources, II. medium I but with $15 \mathrm{mM}$ glucose and $10 \mathrm{mM}$ citric acid (CS: carbon sources), III. a mix of the spent medium of a given partner species $(50 \%)$ and medium I (50\% of a $2 \times$ concentrated solution), IV. the spent medium of a partner species, V. a mix of the spent medium of a partner species $(50 \%)$ and medium II (50\% of a $2 \times$ concentrated solution). Square colors reflect area under the growth curve (AUC) under these different conditions relative to the AUC in conditions I and II (gradient legend). See Fig. S2 for model growth curves that generated these. (B) Results of spent media (SM) assays, where each row shows how a given species grew normalized by conditions I and II in the different SM conditions (III, IV, V) for each SM producer (see Fig. S3 for growth curves and Fig. S4 for AUCs with statistical comparisons). Comparing these patterns to the predictions in panel A determined the hypothesized interactions. When $C t$ was grown in the spent media of others, interactions did not qualitatively match any predicted scenarios. The growth of $M s$ is not shown here, as the OD data and the CFUs data were not coherent. (C) Consumption [\%] of glucose and citric acid by the 4 species based on commercial chemical kits applied to the spent media in stationary phase (72 and 96 hours of growth). We also analyzed the SM of Ct after growing in the pure SM of At and Ms (samples collected at the end of the SM assay after $\sim 64$ hours, thus labelled "end SMA"). (D) Growth curves of $C t$ as $\mathrm{OD}_{600}$ over time when grown in the five culture conditions, with either $A t$ or Ms generating the spent media, $(\mathrm{n}=3$, $\pm \mathrm{sd}$, error bars are present but very small). Roman numerals indicate culture conditions as in panel A, colours indicate spent media producing species.

which carbon sources the four species use (see Methods). We ${ }_{195}$ found that $A t$ consumes mostly glucose, $C t$ mostly citric acid, 196 Oa consumes both, while $M s$ consumes little of either (Fig. 197 1C). In agreement with this, $A t$ reflects the niche separation ${ }_{198}$ model in the spent media of $C t$ and $M s$. Since $A t$ does not ${ }_{199}$ consume citric acid, but overlaps with $\mathrm{Oa}$ in consuming glu- 200 cose, SM interactions of both $A t$ and $O a$ lie in between ex- 201 ploitative competition and niche separation. Similarly, in the 202 SM of $C t, O a$ follows a pattern in between exploitative com- 203 petition and niche separation. We observed some evidence ${ }_{204}$ for cross-feeding from $\mathrm{Ms}$ to $\mathrm{Oa}$, and for interference com-

petition from $\mathrm{Ct}$ to $\mathrm{Oa}$ (see Fig. S4 for statistics), but do not 205 explore this further. Instead, we focus on why $C t$ did not fit 206 our model's predictions.

\section{$C t$ has a shorter lag phase in the pure spent media ${ }_{209}$ of $\boldsymbol{A t}$ and $\boldsymbol{M s}$ but grows poorly in all other conditions. 210}

When $C t$ grows in the SM of either At or $M s$ (condition IV), ${ }_{211}$ its AUC is significantly higher (t-test with Bonferroni correc- 212 tion, both $d f=5, P<0.001)$ than in II, which is due to a ${ }_{213}$ much shorter lag phase (Fig. 1D). However, when we replen- 214 ished the growth medium (condition V), $C t$ grew significantly ${ }_{215}$ worse than in the original medium (condition II) (both $d f=5,{ }_{216}$ $P<0.001$, Fig. 1B, D). We observe the same pattern in its ${ }_{217}$ own and in $O a$ 's SM (growth in $\mathrm{V}$ is significantly worse than ${ }_{218}$ II, $P<0.001$, Fig. S4). This is surprising because condition ${ }_{219}$ $\mathrm{V}$ should contain at least the same concentration of carbon 220 sources as in condition II.

This led us to suspect that some of the replenished compounds might impair the growth of $C t$ because they end up at higher concentrations than in the original medium. Accordingly, we tested a first hypothesis: that one or more of the compounds in the NC medium inhibit the growth of $\mathrm{Ct}$, while $A t$ and $M s$ are able to reduce their concentration and thereby shorten the lag phase of $C t$. According to our model, such "cross-detoxification" could be a valid explanation for the observed patterns (Fig. S6).

\section{No-carbon compounds delay the growth of $\mathrm{Ct}$ but are} not reduced by $\boldsymbol{A t}$ or Ms. To explore whether the NC medium could affect the growth of $C t$, we first manipulated its two main ingredients: M9 and Hutner's vitamin free Mineral Base (HMB, see Methods). While reducing the concentration of HMB had no positive effect (Fig. S7), a small decrease in the concentration of M9 shortened the lag phase of $\mathrm{Ct}$, while reducing it further had a detrimental effect (Fig. 2A, see Fig. S8A for CFUs). As changing the concentration of M9 changes both $\mathrm{pH}$ and osmolarity simultaneously, we next tested the effect of each separately and found that both (i) increasing the $\mathrm{pH}$ and (ii) decreasing $\mathrm{M} 9$ but keeping the $\mathrm{pH}$ constant (lowering osmolarity) shortened the lag phase of $C t$ (Fig. 2B). To assess if specific compounds in the M9 (see Methods) could influence the lag phase of $C t$, we tested the effect of each compound on the growth of $C t$ 
by decreasing their concentration in the NC (Fig. 2C). Of 268 all the compounds, we found that the total concentration of 269 phosphate $\left(\mathrm{Na}_{2} \mathrm{HPO}_{4}\right.$ and $\left.\mathrm{KH}_{2} \mathrm{PO}_{4}\right)$ was the only one that 270 influenced the lag phase of $C t$ independently of the pH (Fig. 271 2D). Changing the ratio of the two ions $\left(\mathrm{Na}^{+}\right.$and $\left.\mathrm{K}^{+}\right)$by ${ }_{272}$ changing the ratio of the corresponding salts $\left(\mathrm{Na}_{2} \mathrm{HPO}_{4}\right.$ and ${ }_{273}$ $\mathrm{KH}_{2} \mathrm{PO}_{4}$ ) also affected the lag phase: a higher proportion of ${ }_{274}$ potassium shortened it while sodium increased it (Fig. 2E). 275 These findings suggest that the $\mathrm{NC}$ medium is suboptimal for 276 $C t$ and lengthens its lag phase: a controlled $\mathrm{pH}$, a lower phos- 277 phate concentration, or a smaller sodium to potassium ratio 278 in the M9 allow $C t$ to grow earlier. Accordingly, our most 279 parsimonious explanation for its SM behavior (Fig. 1B) was 280 that $A t$ and $M s$ can modify at least one of those factors in the 281 medium. However, when we measured $\mathrm{pH}$, osmolarity, and 282 the concentration of phosphate, and sodium and potassium 283 ions in the SM of the 4 species (Fig. S9), we found that none 284 differed between the original MM and the SM of $A t$ and $M s{ }_{285}$ in a way that would explain the shortened lag phase of Ct. ${ }^{286}$ In sum, several properties of the NC medium lengthen the ${ }^{287}$ lag phase of $C t$, but $A t$ and $M s$ appear unable to significantly ${ }^{288}$ modify these properties in a way that would explain why $C t^{289}$ grows so well in their SM. We therefore rejected our first 290 hypothesis.

$\boldsymbol{C t}$ feeds on metabolites produced by $\boldsymbol{A t}$ and $\mathbf{M s}$. An- ${ }^{293}$ other hypothesis that could explain the shortened lag phase ${ }^{294}$ of $C t$ in the SM of $A t$ and $M s$ is that their SM contains ${ }^{295}$ metabolic by-products that allow $C t$ to grow earlier. To find ${ }^{296}$ such candidate molecules, we performed an untargeted Liquid Chromatography - Mass Spectrometry (LC-MS) analysis ${ }^{297}$ (see Methods) on the SM of $A t, C t$ and $M s$ and on the $\mathrm{SM}_{298}$ of $A t$ and $M s$ after $C t$ had grown in it to assess if $A t$ and $M s_{299}$ secrete molecules that $C t$ then consumes (Fig. 3A, full list in ${ }_{300}$ Fig. S10).

We identified several compounds that follow this pattern (Fig. 302 $3 \mathrm{~A}$ ) and selected three - based on availability, cost and ease ${ }_{303}$ of use - to assess their effect on the lag phase of $C t$ : hypoxan- 304 thine, oxoglutarate and proline (Fig. 3B). As the LC-MS only ${ }_{305}$ yielded the relative abundances of each compound, we added 306 several concentrations to $C t$ growing in MM. We found that 307 a range of concentrations shortens the lag phase of $C t$ signif- $з о 8$ icantly (Oxoglutarate: from $0.39 \mathrm{mM}$ to $12.5 \mathrm{mM}$, table $\mathrm{S} 1 ; 309$ proline: $\geq 0.39 \mathrm{mM}$, table $\mathrm{S} 2$; hypoxanthine: $\geq 0.62 \mathrm{mM}$, ta- 310 ble S3). At high enough concentrations, all three metabolites 311 also increased the final yield of $C t$, suggesting that they act ${ }_{312}$ as carbon sources (Oxoglutarate: from $0.09 \mathrm{mM}$ to $12.5 \mathrm{mM}$, 313 table S4; proline: $\geq 0.78 \mathrm{mM}$, table S5; hypoxanthine: $\geq_{314}$
$1.25 \mathrm{mM}$, table S6). At its two highest concentrations (25 and $50 \mathrm{mM}$ ), oxoglutarate even had an inhibitory effect on $C t$. To confirm that these three metabolites could act as carbon sources, we cultured $C t$ in NC medium containing each of the metabolites at an intermediate concentration (oxoglutarate and proline: $1.56 \mathrm{mM}$; hypoxanthine: $1.25 \mathrm{mM}$ ) as the sole carbon source and observed significant growth in all three cases (Fig. 3C).

It appears then that these three metabolites are being crossfed by $C t$. If they are responsible for the shortening of the lag phase of $C t$ in the SM of $A t$ and $M s$, the replenishment of the medium should cancel this effect like we observed in the spent media assays (Condition III, V, Fig. 1B, D). We grew $C t$ in MM supplemented with each of the three metabolites (at concentrations based on Fig. 3B) and added increasing concentrations of the NC medium (Fig. 3D). As in our original experiments, the growth of $C t$ was impaired despite the presence of the metabolites and the original carbon sources in all conditions compared to the positive controls (no addition of $\mathrm{NC}$ or metabolites), supporting our hypothesis. These results are in line with our updated model (Fig. S6) that includes the inhibitory effect of the medium.

Overall, we find that different properties of the NC compounds can lengthen the time $C t$ takes to start growing. Metabolites secreted by At and $M s$ - at least the three that we tested - are able to reduce this effect through cross-feeding. However, when the concentration of NC compounds is high enough, the effect of the metabolites is insufficient and $C t$ grows very little on the timescale of our experiments.

\section{Discussion}

Before running our SM assays, we used a simple mathematical model to generate our base expectations under different experimental conditions. We were surprised when the behavior of one species, $\mathrm{Ct}$, did not correspond to the predictions of any of the scenarios simulated by the model. Our further analysis revealed that this was because our model assumed that the growth medium could either allow cells to grow or not, but it could not inhibit species, such that increasing its concentration would delay their growth. In hindsight, we know that designing growth media for different species can be quite challenging $(32,33)$, so we might expect negative effects of some media on some bacterial species.

Based on this new intuition, we updated our model to cover cases when the medium inhibits growth (Fig. 4A, Fig. S6). The patterns in this new model fit qualitatively with what we observed for $C t$, but also drew our attention to another issue: it was challenging to distinguish between cross-feeding and 

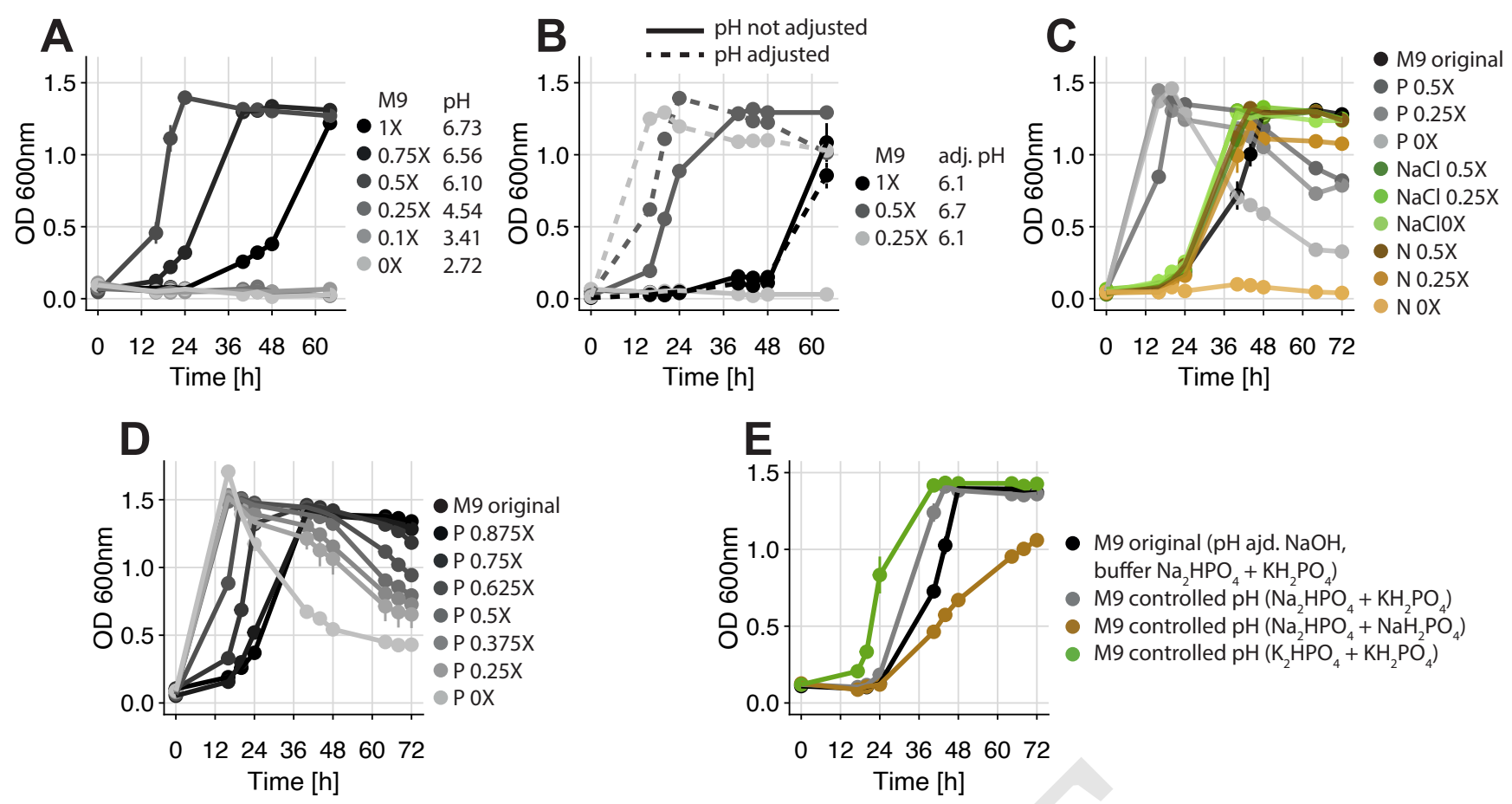

Fig. 2. Growth of $C t$ (as $\mathrm{OD}_{600}$ ) in minimal medium (MM) made with no-carbon (NC) medium where we modified the M9 part. (A) Growth in $M M$ with decreasing concentrations of M9. The resulting $\mathrm{pH}$ is indicated for each fresh MM, showing that decreasing the concentration of $\mathrm{M} 9$ also decreases the $\mathrm{pH}$. (B) Growth in $\mathrm{MM}$ with decreasing concentrations of $\mathrm{M} 9$ with and without manual $\mathrm{pH}$ adjustment to either 6.1 or 6.7 using $\mathrm{NaOH}$. Decreasing the concentration of $\mathrm{M} 9$ while keeping a pH close to neutrality shortens the lag phase of $C t$. (C) Growth in MM with M9 that vary in the concentration of its main components: phosphate (overall $\mathrm{Na}_{2} \mathrm{HPO}_{4} \mathrm{plus}^{\mathrm{K}} \mathrm{KH}_{2} \mathrm{PO}_{4}$ concentration, abbreviated $\mathrm{P}$ ), sodium chloride $(\mathrm{NaCl})$ and nitrogen $\left(\mathrm{NH}_{4} \mathrm{Cl}\right.$, abbreviated $\left.\mathrm{N}\right)$. The $\mathrm{pH}$ is adjusted to $6.7 \mathrm{with} \mathrm{NaOH}$ in all conditions ( $\mathrm{pH}$ of the original $\mathrm{MM}$ ). The overall concentration of phosphate seems to be the sole factor that affects Ct's lag phase (D) Growth in MM with M9 varying in its overall concentration of phosphate $\left(\mathrm{Na}_{2} \mathrm{HPO}_{4}\right.$ plus $\mathrm{KH}_{2} \mathrm{PO}_{4}$, abbreviated $\mathrm{P}$ ). The $\mathrm{pH}$ is adjusted to 6.7 with $\mathrm{NaOH}$ in all conditions ( $\mathrm{pH}$ of the original $\left.\mathrm{MM}\right)$. (E) Growth in MM with $\mathrm{M9}$ that have either phosphate compounds comprising only $\mathrm{Na}^{+}$ions $\left(\mathrm{Na}_{2} \mathrm{HPO}_{4}\right.$ plus $\left.\mathrm{NaH}_{2} \mathrm{PO}_{4}\right)$, only $\mathrm{K}^{+}$ions $\left(\mathrm{K}_{2} \mathrm{HPO}_{4}\right.$ plus $\left.\mathrm{KH}_{2} \mathrm{PO}_{4}\right)$ or both ions $\left(\mathrm{Na}_{2} \mathrm{HPO}_{4}\right.$ plus $\left.\mathrm{KH}_{2} \mathrm{PO}_{4}\right)$ but with adjusted ratios so that the $\mathrm{pH}$ is at 6.7 ("controlled $\mathrm{pH}$ ") with no $\mathrm{NaOH}$ adjustment. For all graphs: mean is plotted $\pm \mathrm{sd}, \mathrm{n}=3$.

cross-detoxification. And even though cross-detoxification 336 seemed the more parsimonious explanation, all our experi- 337 ments led us to reject it as the underlying interaction. The ${ }_{338}$ new model does, however, give two clues to distinguish these ${ }_{3 з 9}$ two types of positive interaction without chemical analyses: 340 First, in the pure spent media (condition IV), the final yield ${ }_{341}$ in a cross-detoxification interaction will never exceed that of ${ }_{342}$ the original growth medium (under the assumption that inhi- ${ }_{343}$ bition slows down growth rather than increasing death), while 344 this should be the case for cross-feeding (right arrow in Fig. 345 4B, C, S6); second, in cross-feeding, one may detect a first 346 short stationary phase as cells switch their metabolism to con- 347 sume the second carbon source (left arrow in 4B). We reran ${ }_{348}$ our original experiment in a plate reader to obtain higher time ${ }_{349}$ resolution, and indeed found a significant difference in the ${ }_{350}$ final yield of $C t$ in MM and the SM (condition IV) of $A t_{351}$ $(P<0.01)$ and $M s(P<0.001)$, as well as a small "bump" ${ }_{352}$ at the beginning of the SM growth curves (arrows in Fig. 4D, ${ }_{353}$ Fig. S11, see tables S7 and S8 for statistics on the final yield ${ }_{354}$ and the length of the lag phase, respectively).

355 Our simple spent media assays and updated model can in 356 principle suffice to classify the dominant interactions, at least in defined medium with few carbon sources. They are more powerful than direct co-culture experiments, where the directionality of interactions is obscured. In Fig. S12, we have constructed a decision tree that allows one to classify data using the 5 spent media conditions outlined here, assuming that each species consumes a single carbon source, regardless of how many are present. This is because for simplicity, the medium in our model only includes a single carbon source (it can be extended). We therefore do not apply the decision tree to interactions with $O a$, which we classified based on our knowledge that it consumes both carbon sources.

Even in the simple scenario we have studied, we may be missing additional layers of interactions between these four species. First, all three SM metabolites we tested turned out to be cross-fed, suggesting that others may have a similar effect. This aligns with previous research suggesting that crossfeeding might be quite common $(34,35)$, particularly for species that are in a sub-optimal environment that contains few carbon sources $(36,37)$, or that is toxic (31). It may be then, that the difficulty of finding a growth medium that can 
bioRxiv preprint doi: https://doi.org/10.1101/2022.03.02.482509; this version posted March 2, 2022. The copyright holder for this preprint (which was not certified by peer review) is the author/funder, who has granted bioRxiv a license to display the preprint in perpetuity. It is made available under aCC-BY 4.0 International license.

A

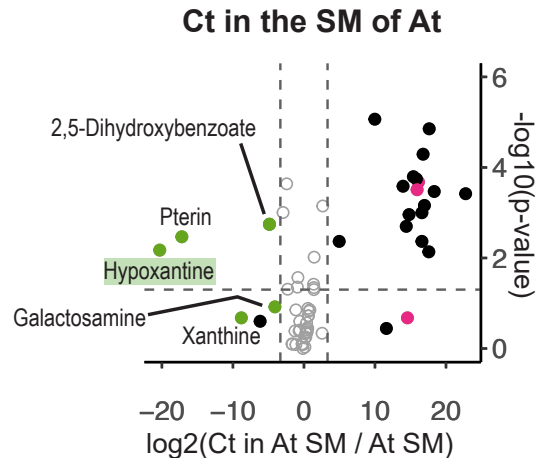

Ct in the SM of Ms

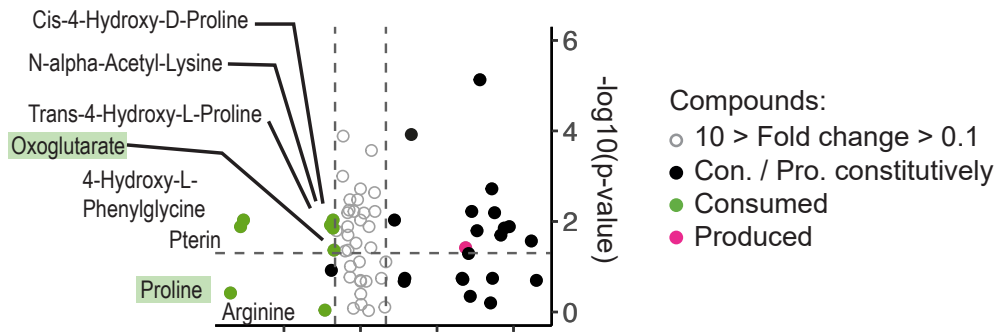

$\begin{array}{llll}-10 & 0 & 10 & 20\end{array}$

$\log 2(\mathrm{Ct}$ in Ms SM / Ms SM)
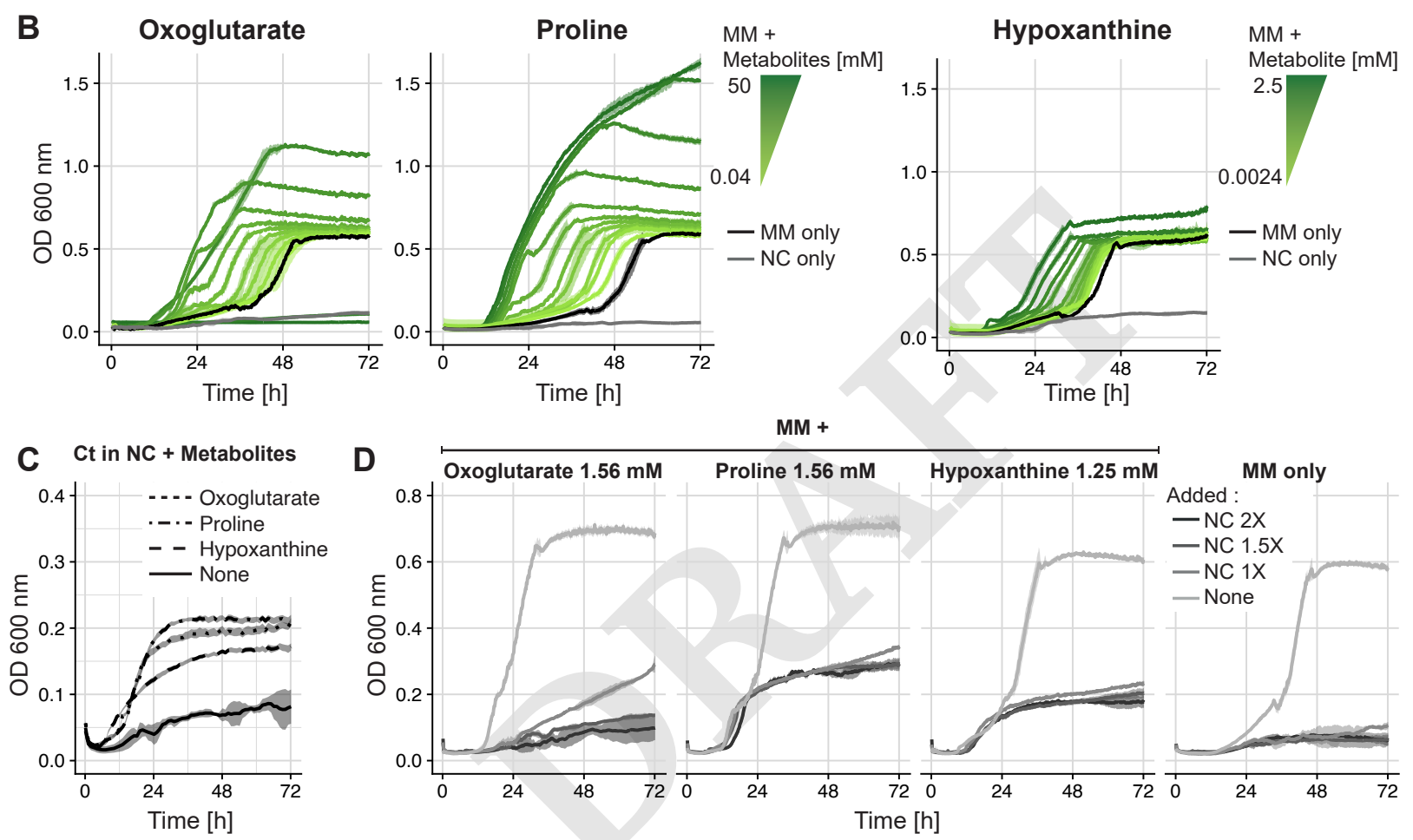

Fig. 3. Identification of three cross-fed metabolites (oxoglutarate, proline, hypoxanthine) and their effect on Ct. (A) Untargeted metabolomics analysis (focusing on polar molecules) performed on the SM of $A t, C t$ and $M s$ and on the SM of $A t$ and $M s$ after $C t$ grew in them to identify metabolites that were produced by $A t$ and $M s$ to then be consumed by $C t$ (relative quantification). Left: X-axis shows the $\log _{2}$ of the ratio (fold change) between the abundance of each metabolite in the spent media of $A t$ after the growth of $C t$ to the abundance of each metabolites in the spent media of At. If metabolites are on the left of the graph (negative), Ct consumed them from At's SM, while if they are on the right (positive) $C t$ produced them. Grey metabolites between the dashed lines have a fold change that is $<10 \times$, which we did not consider. Y-axis shows the $\log _{10}$ of the $p$-value, the 0.05 significance threshold is represented by a dashed line. Metabolites in green boxes were chosen for further analysis. Right: Same as the left panel but for the SM of Ms. In both panels, $\mathrm{n}=3$. ANOVA one-factor (on log10 transformed data) was used to test the significance of metabolite changes in the different conditions. (B) Effect of oxoglutarate, proline and hypoxanthine on the growth of $C t$ in a range of concentrations added to MM, measured as the OD 600 over 72 hours. The mean is plotted and the transparent area around the curves represents the standard deviations. As a control, Ct was grown in MM or NC, ( $\mathrm{n}=4$ ). (C) Effect of $1.56 \mathrm{mM}$ oxoglutarate, $1.56 \mathrm{mM}$ proline and $1.25 \mathrm{mM}$ hypoxanthine on the growth of $\mathrm{Ct}$ in NC medium only, with NC alone as a control over 72 hours. The OD was measured every 10 minutes, the mean is plotted and the transparent area around the curves represents the standard deviations, $(n=3)$. The scale of the $y$-axis is smaller that in panel $D$ to better show the growth curves. (D) Effect of intermediate concentrations of each metabolite in MM with increasing concentrations of NC $(50 \%$ replenishment in either NC medium $1 \times, 1.5 \times$, or $2 \times)$, measured as the $\mathrm{OD}_{600}$ over 72 hours. The mean is plotted and the transparent area around the curves represents the standard deviations. As a control, $C t$ was grown in MM (in the same conditions, $(n=3)$. The data show that all three metabolites could act as carbon sources, could short Ct's lag phase and the effect was reversed on the addition of NC. 


\begin{tabular}{l} 
A Interaction types \\
\begin{tabular}{|l|l|}
\hline Exploitative competition & $\mathrm{EC}$ \\
Exploitative + & interference competition \\
Exploitative + cross-feeding & $\mathrm{EC}+\mathrm{IC}$ \\
$\begin{array}{l}\text { Exploitative competition + } \\
\text { cross-detoxification } \\
\text { Niche separation }\end{array}$ & $\mathrm{EC}+\mathrm{CF}$ \\
$\begin{array}{l}\text { Niche separation + } \\
\text { interference competition } \\
\text { Niche separation + } \\
\text { cross-feeding }\end{array}$ & $\mathrm{NS}$ \\
$\begin{array}{l}\text { Niche separation + } \\
\text { cross-detoxification }\end{array}$ & $\mathrm{NS}+\mathrm{IC}$ \\
\hline
\end{tabular} \\
\hline
\end{tabular}

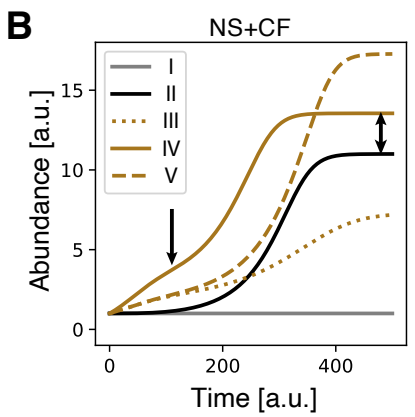

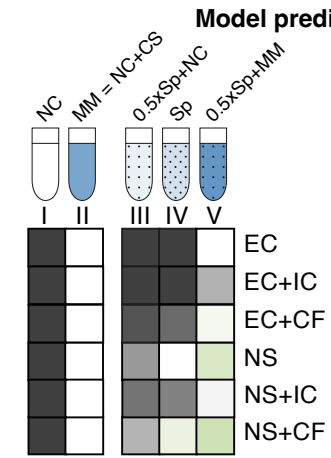

C

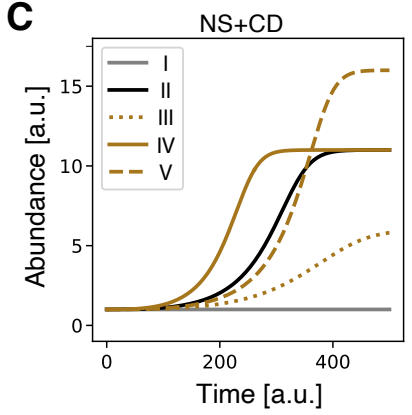

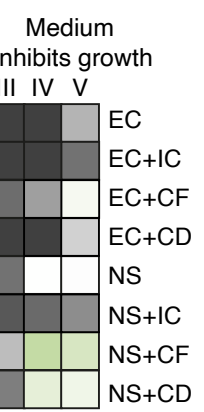
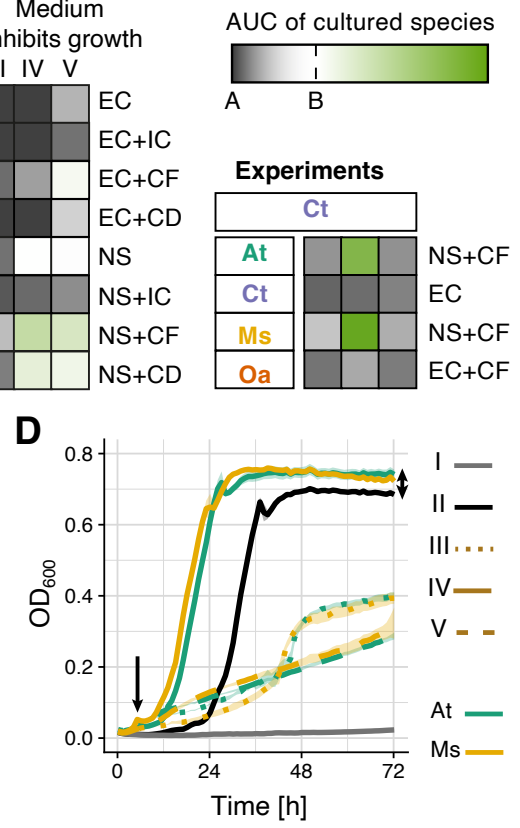

Fig. 4. Revised model better predicts Ct's growth patterns. (A) Model predictions of the original model on the left (same as in Fig. 1A), the model predictions in an environment that inhibits growth in the center and the data for the growth of $C t$ in the SM of the four different species. Although the predictions do not match perfectly, they qualitatively resemble the patterns in the inhibitory growth medium. (B) Model predictions for niche separation + cross-feeding. The single arrow shows the growth "bump" in IV compared to II at around 80 hours due to a switch in carbon sources, and the double arrow a greater final yield in condition IV. (C) Model predictions for niche separation + cross-detoxification. Here, the bump is missing and the final yield is identical in II and IV. (D) Repetition of the experiment shown in Fig. 1D in a 96-well plate with higher time resolution shows the presence of a bump in the two species' SM (condition IV, solid yellow and green lines), indicating a switch in carbon source (see Fig. S11), and a greater final yield compared to growth in fresh medium (condition II, black). Note that the model still does not correspond perfectly to the experimental data, indicating that there may be additional interactions at play.

sustain the individual growth of all members of a community, 380 is precisely what allows us to observe cross-feeding interac- 381 tions. In fact, positive interactions are often "accidental", re- 382 sulting from the secretion of cost-less metabolic by-products 383 by the few species that are able to grow $(8,19,36,38-44)$. 384 While the secretion of metabolites like amino acids by bacte- 385 ria might seem counter-intuitive, several mechanisms like the ${ }_{386}$ maintenance of cell homeostasis (release of over-produced ${ }_{387}$ metabolites) or cell lysis can explain costly metabolic by- 388 product secretion (45). We were also surprised that $M s$ produced several metabo- 390 lites (Fig. 3A) and affected $C t$ 's growth, even though its 391 own population size did not increase significantly (Fig. S5). 392 This aligns with other studies showing that cross-feeding 393 does not require bacterial growth (8). Indeed, the absence of 394 growth does not necessarily indicate metabolic inactivity, as 395 metabolic activity is required to produce enough energy for 396 survival. This suggests that in larger bacterial communities, 397 such as the gut microbiome, non- or slow-growing species 398 should not be ignored, as they may still significantly affect 399 other community members.

400 Despite our efforts, it still remains unclear why the mini- 401 mal medium delays $C t$ 's growth, and why the cross-fed com- 402 pounds allowed it to start growing sooner. One hypothesis is that $C t$ experiences osmotic stress in the minimal medium, which can be reflected in the length of its lag phase $(46,47)$. Given that the other 3 species seemed robust to this stress, the metabolites they secrete could, once consumed, help $C t$ to cope with this stress. Proline, for example, can act as a "compatible solute" (48-51), which are molecules that bacteria synthesize or take up to balance osmotic pressure in hyper-osmotic environments. Alternatively, the metabolites could act as metabolic precursors, allowing $C t$ to synthesize its own compatible solutes de novo. This may be the case for oxoglutarate, which is a direct intermediate in the Krebs cycle (52). In E. coli, oxoglutarate is taken up from the environment (53), but is also leaked (54), hinting that it may be involved in extracellular exchange. Similarly, the purine derivative hypoxanthine is an important nitrogen source and participates in nucleic acid synthesis via the pentose phosphate salvage pathway (55). Interestingly, hypoxanthine was found to mediate interactions influencing biofilm formation between $B$. subtilis and soil bacteria whose cell-free supernatants were analysed similarly to our own approach (HPLC, NMR and HR-MS) (56). Another hypothesis is that $C t$ requires a metabolic shift to grow on citric acid compared to 
other carbon sources, and that increases its lag phase due to 450 a high enzymatic cost $(57,58)$. The presence of metabolites 451 secreted by At and Ms could then allow it to metabolize citric acid more rapidly. Distinguishing between these different ${ }^{452}$ hypotheses could be achieved by engineering $C t$ to report on ${ }^{453}$ osmotic stress, by isotopically labelling the carbon sources 454 and following their metabolic by-products from $A t$ and $M s^{455}$ that are later consumed by $C t$, and/or by testing the role of ${ }^{456}$ the remaining identified metabolites.

The ability to classify interspecies interactions to the level ${ }^{457}$ of distinguishing cross-feeding from cross-detoxification, for ${ }^{458}$ example, is not just a matter of curiosity, but is key to un- ${ }^{459}$ derstanding and predicting community dynamics $(2,59,60){ }^{460}$ Even mechanistic details of cross-feeding can affect commu- ${ }^{461}$ nity dynamics. La Sarre et al. (59) showed that increasing the concentration of a cross-fed metabolite can render it toxic to the partner species, leading to a new community equilibrium. To make matters even more complicated, each species pair is likely to interact in more than one way, but the effects we observe are cumulative (60). Here, we showed how Ms, for ${ }^{46}$ example, produced a whole series of compounds, and that $C t^{467}$ could feed on the two that we tested. But it may well be that ${ }^{46}$ other compounds have small inhibitory effects on $C t$, and that ${ }^{460}$ changing the environmental conditions could increase their ${ }^{470}$ production or leakage rates and alter community dynamics (59).

In conclusion, our work proposes that carefully designed spent media assays together with a simple mathematical model can help to map out the dominant metabolic interactions in more detail than simply labeling them as positive or negative. We have showcased this using a small, synthetic bacterial community in a defined medium, in which we could verify the read-outs from the growth curves with more detailed analyses. It remains to be seen whether our approach ${ }^{40}$ would scale up to more high-throughput approaches in larger communities (as in (36), e.g.) and more complex environments. But ultimately, such simple experimental approaches are needed to predict the dynamics of natural microbial com- ${ }^{484}$ munities.

\section{Material and methods}

Cell culture preparation. Species were grown in monoculture

in TSB (tryptic soy broth) overnight $\left(28^{\circ} \mathrm{C}\right.$, shaking $\left.200 \mathrm{rpm}\right) 489$ and were then diluted to an $\mathrm{OD}_{600}$ of 0.05 in fresh TSB 490 and incubated again for 3 hours in order to reach exponen- 491 tial growth. Each culture was then washed 2 times in PBS 492 (phosphate buffered saline) (centrifugation: $15 \mathrm{~min}$ at 4'000 ${ }_{493}$ rpm, room temperature) and the final bacterial pellets were 494 resuspended in the adequate medium so that the initial $\mathrm{OD}_{600}$ would be 0.1 .

Colony forming units (CFUs) measurement. To measure the CFUs, we sampled $20 \mu \mathrm{L}$ of our cultures and diluted them in $180 \mu \mathrm{L}$ of PBS in 96-well plates and proceeded to 10 -fold dilutions down to $10^{-7}$. The dilutions were plated on TSA (tryptic soy agar) as drops and were then spread into lines.

Media recipes. For the compositions of the different solutions used, see the following tables: minimal medium (MM) - table S9; no-carbon (NC) medium - table S10; HMB - table S11; Metal 44 - table S12; M9 - table S13; M9 with controlled pH used in Fig. 2E - tables S14, S15, S16.

Spent media (SM) assays. Spent medium (SM) from each of the four species were obtained by growing them in large volumes $(\mathrm{V}=30 \mathrm{~mL})$ in $\mathrm{MM}$ until they reached stationary phase ( $\sim 72 \mathrm{~h}$ to 96 hours, decided by $\mathrm{OD}_{600}$ determination). We then centrifuged the bacterial culture $\left(20 \mathrm{~min}, 4^{\prime} 000 \mathrm{rpm}\right.$, room temperature) and collected the supernatants. We centrifuged the supernatants again to eliminate as many bacterial cells and debris as possible before filtering them using vacuum filters (TPP vacuum filtration "rapid"-Filtermax, membrane: PES, $0.22 \mu \mathrm{m})$. From those SM we prepared 3 media conditions to test the effect of the SM on our 4 species: conditions C (SM:NC $2 \times=1: 1$ ), condition D (pure SM only) and condition $\mathrm{E}$ (SM:MM $2 \times=1: 1)$. Our control conditions were fresh $\mathrm{NC}$ medium (A, negative control) and fresh MM (B, positive control). We grew the 4 species in mono-cultures in those 5 conditions $(\mathrm{V}=4 \mathrm{~mL})$ over 60 hours and measured the $\mathrm{OD}_{600}$ over time (using Ultrospec 10 cell density meter, Biochrom) and performed CFU counts before the initial incubation, at $24 \mathrm{~h}$ and $48 \mathrm{~h}$. We calculated the area under the $\mathrm{OD}_{600}$ growth curves (AUC) and used this value as a proxy for growth (Andri et mult. al. S (2021). DescTools: Tools for Descriptive Statistics. R package version 0.99.44) (R version 4.1.2). We repeated those SM assays in 96-well plates ( $\mathrm{V}=$ $200 \mu \mathrm{L}$ ) to increase the resolution of our data and measured the $\mathrm{OD}_{600}$ every 10 minutes for 72 hours using a microplate reader (Biotek synergy $\mathrm{H} 1,28^{\circ} \mathrm{C}$, continuous double-orbital shaking).

Glucose, citric acid, phosphate, sodium, potassium and osmolarity quantification. To quantify glucose, citric acid, phosphate, sodium and potassium in the SM of $A t, C t$ and $M s$ we used different chemical kits. We generated SM for each species as described previously (section "Spent media (SM) assays", total incubation time $\simeq 89$ hours) and followed 
the specific protocols of each kit to determine which con- 542 centration of SM to test given the theoreticalconcentration of ${ }_{543}$ the tested compounds in fresh MM. Glucose kit: Glucose ${ }_{544}$ (HK) Assay Kit (Sigma, product Code GAHK-20). Citric ${ }_{545}$ acid kit: Citric Acid Assay Kit (Megazyme, product Code: 546 K-CITR). Phosphate kit: Phosphate Assay Kit (Colorimet- 547 ric) (Abcam, product code: ab65622). Sodium kit: Sodium ${ }_{548}$ Assay Kit (Colorimetric) (Sigma, product code: MAK247). 549 Potassium kit: Potassium Assay Kit (Fluorometric) (Ab- 550 cam, product code: ab252904). Osmolarity was measured ${ }_{551}$ in each SM sample using an osmometer (Osmomat 030 by 552 Gonotec).

\section{Metabolomics analyses of SM samples. Untargeted} metabolomics analyses were performed for us at Metabolomics Platform, Faculty of Biology and Medicine, University of Lausanne, on the following samples, focusing on polar (water soluble) compounds : fresh minimal medium (MM); SM of At, Ct and $M s$ (generated as described in "Spent media (SM) assays") and the SM of $A t$ and $M s$ after $C t$ grew in them (total incubation time $\simeq 60$ hours). To summarize the procedure, we first identified which ${ }^{56}$ metabolites were produced by $A t, C t$ and $M s$ when grown ${ }^{562}$ in mono-culture in MM (fresh MM compared to the SM of ${ }^{56}$ $A t, C t$ and $M s$ ), and then compared this list of metabolites ${ }^{56}$ to the ones identified in the SM of $A t / M s$ after $C t$ grew in ${ }^{565}$ them (SM of $A t$ and $M s$ compared to the SM of $A t$ and $M s^{566}$ after $C t$ grew in them). Using those comparisons we could ${ }^{56}$ identify 64 compounds that were produced by $A t$ and $M s^{566}$ and then consumed by $C t$ or that were absent in the SM of $A t$ and $M s$ but were later produced by $C t$. From that list we only considered the compounds consumed by $\mathrm{Ct}$ with a fold change of at least 10. Further details are in Supplementary ${ }^{57}$ note S2.

Testing the effect of metabolites on Ct: oxoglutarate, proline 574 and hypoxanthine. We followed a similar protocol for all the 575 compounds. For oxoglutarate and proline, the same concen- 576 tration was tested. In a $96-$ well plate, we added $180 \mu \mathrm{L}$ of 577 water in wells B-E(1) (4 replicates). We then added $20 \mu \mathrm{L}_{578}$ of a $1 \mathrm{M}$ stock solution of either oxoglutarate or proline so 579 that those wells contained $100 \mathrm{mM}$ of the metabolite tested. 580 The other wells in lines B to E were filled with $100 \mu \mathrm{L}$ of wa- 581 ter. Serial dilutions from wells B-E(1) to B-E(11) were per- 582 formed by transferring $100 \mu \mathrm{L}$ each time ( $2 \times$ dilutions). This ${ }_{583}$ way we obtained 11 concentrations to test on $C t$ (from $50 \mathrm{mM}_{584}$ to $0.04 \mathrm{mM}$ ), in 4 replicates. To those wells, we then added 585 $100 \mu \mathrm{L}$ of $C t$ cultures that were in MM $2 \times$ concentrated and 586 with an OD of 0.2 (following the method described in "Cell 587 culture preparations"). This way, the final concentration of MM is $1 \times$ and the final OD is 0.1 (as usually tested). As controls, we grew $C t$ in MM (in 4 wells we mixed $100 \mu \mathrm{L}$ of water to $100 \mu \mathrm{L}$ of $C t$ culture (in MM $2 \times, \mathrm{OD}=0.2$ )) in addition to $C t$ in NC medium (in 4 wells we mixed $100 \mu \mathrm{L}$ of water to $100 \mu \mathrm{L}$ of $C t$ culture (in NC $2 \times, \mathrm{OD}=0.2$ )). For $h y$ poxanthine, we proceeded slightly differently as its solubility is a lot lower than for the two other metabolites. We prepared a hypoxanthine stock at $5 \mathrm{mM}$ and directly added $200 \mu \mathrm{L}$ to wells B-E(1). We then followed the same logic as for oxoglutarate and proline. We could test concentrations from $2.5 \mathrm{mM}$ to $0.002 \mathrm{mM}$. The growth was assessed by measuring the $\mathrm{OD}_{600}$ every 10 minutes for 72 hours using a microplate reader (Biotek synergy $\mathrm{H} 1,28^{\circ} \mathrm{C}$, continuous double-orbital shaking). The effect of each metabolites on the lag phase of $C t$ and on its final yield compared to both parameters in MM (positive controls) was assessed statistically, see tables S1, S2, S3, S4, S5, S6 (R version 4.1.2).

Testing oxoglutarate, proline and hypoxanthine as carbon sources (fig.3D). To test if the metabolites alone could support the growth of $C t$, we grew $C t$ in $\mathrm{NC}$ medium containing intermediate concentrations of the metabolites (oxoglutarate and proline: $1.56 \mathrm{mM}$; hypoxanthine: $1.25 \mathrm{mM}$ ). We prepared $\mathrm{Ct}$ cultures (following the method described in "Cell culture preparations") with an OD of 0.2 in water and in a 96-well plate mixed $100 \mu \mathrm{L}$ of culture to $100 \mu \mathrm{L} \mathrm{NC}+$ metabolite $2 \times$. The growth was assessed by measuring the $\mathrm{OD}_{600}$ every 10 minutes for 72 hours using a microplate reader (Biotek synergy $\mathrm{H} 1,28^{\circ} \mathrm{C}$, continuous double-orbital shaking).

Testing oxoglutarate, proline and hypoxanthine with increasing concentration of NC compounds on Ct. To test the effect of the metabolites on $C t$ when increasing concentrations of $\mathrm{NC}$ compounds are added, we chose an intermediate concentration of each metabolites (oxoglutarate and proline: $1.56 \mathrm{mM}$; hypoxanthine: $1.25 \mathrm{mM}$ ). We tested the growth of $C t$ in $\mathrm{MM}+$ metabolite when adding $\mathrm{NC}$ medium $2 \times, 1.5 \times$ or $1 \times$. We thus prepared $C t$ cultures in $\mathrm{MM}+$ metabolites $2 \mathrm{X}$ with an OD of 0.2 and one culture of $C t$ in MM $2 \mathrm{X}$ only (as control). In a 96 well-plate, we added $100 \mu \mathrm{L}$ of $C t$ culture to $100 \mu \mathrm{L}$ of either NC $2 \times, 1.5 \times, 1 \times$ or water (as control). This way the MM is $1 \times$ concentrated and $C t$ is at an OD of 0.1 (as usually tested). As a negative control we also grew $C t$ in $\mathrm{NC}$ medium $(\mathrm{OD}=0.1)$. The growth was assessed by measuring the $\mathrm{OD}_{600}$ every 10 minutes for 72 hours using a microplate reader (Biotek synergy $\mathrm{H} 1,28^{\circ} \mathrm{C}$, continuous double-orbital shaking). 
bioRxiv preprint doi: https://doi.org/10.1101/2022.03.02.482509; this version posted March 2, 2022. The copyright holder for this preprint (which was not certified by peer review) is the author/funder, who has granted bioRxiv a license to display the preprint in perpetuity. It is made available under aCC-BY 4.0 International license.

Mathematical model. The mathematical model is described in 654 supplementary note S3.

\section{ACKNOWLEDGEMENTS}

We thank Christoph Keel, Rizlan Bernier-Latmani, Björn Vessman, Sho Shibasaki, Aurore Picot and Afra Salazar for very useful and constructive feedback on the manuscript. Untargeted metabolomic profiling was performed at 661 the Metabolomics Platform, Faculty of Biology and Medicine, University of Lau- 662 sanne. We acknowledge the entire team for their work from sample prepara- 663 tion and data acquisition to data processing and metabolite identification. We 664 particularly thank Julijana Ivanisevic and Hector Gallart-Ayala for discussing the 665 analyses with us. We also thank Alice Wallef, Nastassia Quévit for additional 666 experiments, and Gwenaël Labouebe for access to the osmometer. We thank 667 Björn Vessman for developing the first version of the mathematical model code 668 https://gitlab.com/eccemic/facilitation2019. A.R.D.S. is funded 669 by Swiss National Science Foundation grant PCEGP3_181272, R.D.M. by Euro- 670 pean Research Council grant 715097, S. T. by the NCCR Microbiomes, and S.M. 671 by all three grants.

\section{References} Harcombe, Jorge Peña, Sam P. Brown, and Michael E. Hochberg. Environmentally Medi- 679 ated Social Dilemmas. Trends in Ecology \& Evolution, 34(1):6-18, 2019

2. Babak Momeni, Li Xie, and Wenying Shou. Lotka-Volterra pairwise modeling fails to capture 68 diverse pairwise microbial interactions. eLife, 6:e25051, 2017

3. Nicole A. Abreu and Michiko E. Taga. Decoding molecular interactions in microbial commu- 683 nities. FEMS Microbiology Reviews, 40(5):648-663, 2016.

4. Lori Niehaus, lan Boland, Minghao Liu, Kevin Chen, David Fu, Catherine Henckel, Kaitlin 685 Chaung, Suyen Espinoza Miranda, Samantha Dyckman, Matthew Crum, Sandra Dedrick, 686 Wenying Shou, and Babak Momeni. Microbial coexistence through chemical-mediated in- 687 teractions. Nature Communications, 10(1):2052, 2019.

5. Elena Butaitè, Michael Baumgartner, Stefan Wyder, and Rolf Kümmerli. Siderophore cheat- 689 ing and cheating resistance shape competition for iron in soil and freshwater Pseudomonas 690 communities. Nature Communications, 8(1):414, 2017.

6. Andrés Aranda-Diaz, Benjamin Obadia, Ren Dodge, Tani Thomsen, Zachary F Hallberg, Zehra Tüzün Güvener, William B Ludington, and Kerwyn Casey Huang. Bacterial inter- 693 species interactions modulate $\mathrm{pH}$-mediated antibiotic tolerance. eLife, 9, 2020.

7. Christoph Ratzke and Jeff Gore. Modifying and reacting to the environmental pH can drive 695 bacterial interactions. PLoS biology, 16(3):e2004248, 2018.

8. Ryan K. Fritts, Alexandra L. McCully, and James B. McKinlay. Extracellular Metabolism Sets 697 the Table for Microbial Cross-Feeding. Microbiology and Molecular Biology Reviews, 85(1), 698 2021.

9. Alan R Pacheco and Daniel Segrè. A multidimensional perspective on microbial interac- 700 tions. FEMS Microbiology Letters, 366(11), 2019

10. Melanie Ghoul and Sara Mitri. The Ecology and Evolution of Microbial Competition. Trends 702 in Microbiology, 24(10):833-845, 2016.

11. D. Schluter, T. D. Price, and P. R. Grant. Ecological Character Displacement in Darwin's 704 Finches. Science, 227(4690):1056-1059, 1985.

12. Francesca Fiegna, Alejandra Moreno-Letelier, Thomas Bell, and Timothy G Barraclough. 706 Evolution of species interactions determines microbial community productivity in new envi- 707 ronments. The ISME Journal, 9(5):1235-1245, 2015.

13. Damian W Rivett, Thomas Scheuerl, Christopher T Culbert, Shorok B Mombrikotb, Emma 709 Johnstone, Timothy G Barraclough, and Thomas Bell. Resource-dependent attenuation of 710 species interactions during bacterial succession. The ISME journal, 2016.

14. Abigail I. Pastore, György Barabás, Malyon D. Bimler, Margaret M. Mayfield, and Thomas E. 712 Miller. The evolution of niche overlap and competitive differences. Nature Ecology and 713 Evolution, 5(3):330-337, 2021.

15. Elisa T. Granato, Thomas A. Meiller-Legrand, and Kevin R. Foster. The Evolution and Ecol- 715 ogy of Bacterial Warfare. Current Biology, 29(11):R521-R537, 2019.

16. Karen De Roy, Massimo Marzorati, Pieter Van den Abbeele, Tom Van de Wiele, and Nico 717 Boon. Synthetic microbial ecosystems: an exciting tool to understand and apply microbial 718 communities. Environmental Microbiology, 16(6):1472-1481, 2014.

17. Tobias Großkopf and Orkun S. Soyer. Synthetic microbial communities. Current Opinion in 720 Microbiology, 18(1):72-77, 2014.

18. Ophelia S Venturelli, Alex C Carr, Garth Fisher, Ryan H Hsu, Rebecca Lau, Benjamin P 722 Bowen, Susan Hromada, Trent Northen, and Adam P Arkin. Deciphering microbial interac- 723 tions in synthetic human gut microbiome communities. Molecular Systems Biology, 14(6): 724 e8157, 2018.
19. Olga Ponomarova and Kiran Raosaheb Patil. Metabolic interactions in microbial communities: untangling the Gordian knot. Current Opinion in Microbiology, 27:37-44, 2015.

20. Johannes Cairns, Roosa Jokela, Jenni Hultman, Manu Tamminen, Marko Virta, and Teppo Hiltunen. Construction and Characterization of Synthetic Bacterial Community for Experimental Ecology and Evolution. Frontiers in genetics, 9:312, 2018.

21. William Harcombe. Novel cooperation experimentally evolved between species. Evolution, 64(7):2166-72, 2010

22. Stephen R. Lindemann, Hans C. Bernstein, Hyun Seob Song, Jim K. Fredrickson, Matthew W. Fields, Wenying Shou, David R. Johnson, and Alexander S. Beliaev. Engineering microbial consortia for controllable outputs.

23. Wentao Kong, David R. Meldgin, James J. Collins, and Ting Lu. Designing microbial consortia with defined social interactions. Nature Chemical Biology, 14(8):821-829, 2018.

24. Ryan Tsoi, Zhuojun Dai, and Lingchong You. Emerging strategies for engineering microbial communities. Biotechnology Advances, 37(6):107372, 2019.

25. Marika Ziesack, Travis Gibson, John K W Oliver, Andrew M Shumaker, Bryan B Hsu, David T Riglar, Tobias W Giessen, Nicholas V DiBenedetto, Lynn Bry, Jeffrey C Way, Pamela A Silver, and Georg K Gerber. Engineered Interspecies Amino Acid Cross-Feeding Increases Population Evenness in a Synthetic Bacterial Consortium. mSystems, 4(4), 2019.

26. Alex J.H. Fedorec, Behzad D. Karkaria, Michael Sulu, and Chris P. Barnes. Single strain control of microbial consortia. Nature Communications 2021 12:1, 12(1):1-12, 2021.

27. Sonja Blasche, Yongkyu Kim, Ana Paula Oliveira, and Kiran R. Patil. Model microbial communities for ecosystems biology. Current Opinion in Systems Biology, 6:51-57, 2017.

28. Didier Gonze, Katharine $Z$ Coyte, Leo Lahti, and Karoline Faust. Microbial communities as dynamical systems. Current Opinion in Microbiology, 44:41-49, 2018.

29. Ali R. Zomorrodi and Daniel Segrè. Synthetic Ecology of Microbes: Mathematical Models and Applications. Journal of Molecular Biology, 428(5):837-861, 2016.

30. Maureen A. O'Malley and Emily C. Parke. Microbes, mathematics, and models. Studies in History and Philosophy of Science Part A, 72:1-10, 2018.

31. Philippe Piccardi, Björn Vessman, and Sara Mitri. Toxicity drives facilitation between 4 bacterial species. Proceedings of the National Academy of Sciences of the United States of America, 116(32):15979-15984, 2019.

32. Karsten Zengler, Gerardo Toledo, Michael Rappé, James Elkins, Eric J. Mathur, Jay M. Short, and Martin Keller. Cultivating the uncultured. Proceedings of the National Academy of Sciences, 99(24):15681-15686, 2002.

33. S. S. Epstein. The phenomenon of microbial uncultivability. Current Opinion in Microbiology, 16(5):636-642, 2013.

34. Joshua E Goldford, Nanxi Lu, Djordje Bajić, Sylvie Estrela, Mikhail Tikhonov, Alicia Sanchez-Gorostiaga, Daniel Segrè, Pankaj Mehta, and Alvaro Sanchez. Emergent simplicity in microbial community assembly. Science, 361(6401):469-474, 2018.

35. Samay Pande and Christian Kost. Bacterial Unculturability and the Formation of Intercellular Metabolic Networks. Trends in Microbiology, 25(5):349-361, 2017.

36. Jared Kehe, Anthony Ortiz, Anthony Kulesa, Jeff Gore, Paul C. Blainey, and Jonathan Friedman. Positive interactions are common among culturable bacteria. Science Advances, 7 (45):7159, 2021.

37. Martina Dal Bello, Hyunseok Lee, Akshit Goyal, and Jeff Gore. Resource-diversity relationships in bacterial communities reflect the network structure of microbial metabolism. Nature Ecology \& Evolution 2021 5:10, 5(10):1424-1434, 2021.

38. Alan R. Pacheco, Mauricio Moel, and Daniel Segrè. Costless metabolic secretions as drivers of interspecies interactions in microbial ecosystems. Nature Communications, 10 (1):103, 2019.

39. Glen D'Souza, Shraddha Shitut, Daniel Preussger, Ghada Yousif, Silvio Waschina, and Christian Kost. Ecology and evolution of metabolic cross-feeding interactions in bacteria. Natural Product Reports, 35(5):455-488, 2018.

40. Gregory L. Medlock, Maureen A. Carey, Dennis G. McDuffie, Michael B. Mundy, Natasa Giallourou, Jonathan R. Swann, Glynis L. Kolling, and Jason A. Papin. Inferring Metabolic Mechanisms of Interaction within a Defined Gut Microbiota. Cell Systems, 7(3):245-257, 2018.

41. Emilie E.L. Muller, Karoline Faust, Stefanie Widder, Malte Herold, Susana Martínez Arbas, and Paul Wilmes. Using metabolic networks to resolve ecological properties of microbiomes. Current Opinion in Systems Biology, 8:73-80, 2018.

42. Adam Z Rosenthal, Yutao Qi, Sahand Hormoz, Jin Park, Sophia Hsin-Jung Li, and Michael B Elowitz. Metabolic interactions between dynamic bacterial subpopulations. eLife, 7, 2018.

43. Ali R. Zomorrodi and Daniel Segrè. Intracellular metabolic circuits shape inter-species microbial interactions. bioRxiv, page 127332, 2017.

44. Jeremy M Chacón and William R Harcombe. The Power of Metabolism for Predicting Microbial Community Dynamics. mSystems, 4(3), 2019.

45. Sebastian Gude, Gordon J. Pherribo, and Michiko E. Taga. Emergence of Metabolite Provisioning as a By-Product of Evolved Biological Functions. mSystems, 5(3), 2020.

46. Philip G. Hamill, Andrew Stevenson, Phillip E. McMullan, James P. Williams, Abiann D. R. Lewis, Sudharsan S, Kath E. Stevenson, Keith D. Farnsworth, Galina Khroustalyova, Jon Y. 
bioRxiv preprint doi: https://doi.org/10.1101/2022.03.02.482509; this version posted March 2, 2022. The copyright holder for this preprint (which was not certified by peer review) is the author/funder, who has granted bioRxiv a license to display the preprint in perpetuity. It is made available under aCC-BY 4.0 International license.

Takemoto, John P. Quinn, Alexander Rapoport, and John E. Hallsworth. Microbial lag phase can be indicative of, or independent from, cellular stress. Scientific Reports, 10(1):5948, 2020.

47. Robert L. Bertranda. Lag phase is a dynamic, organized, adaptive, and evolvable period that prepares bacteria for cell division. Journal of Bacteriology, 201(7), 2019.

48. Nuno Empadinhas and Milton S. Da Costa. Osmoadaptation mechanisms in prokaryotes: Distribution of compatible solutes.

49. Janet M. Wood, Erhard Bremer, Laszlo N. Csonka, Reinhard Kraemer, Bert Poolman, Tiemen Van der Heide, and Linda T. Smith. Osmosensing and osmoregulatory compatible solute accumulation by bacteria. In Comparative Biochemistry and Physiology - A Molecular and Integrative Physiology, volume 130, pages 437-460,

50. Roy D. Sleator and Colin Hill. Bacterial osmoadaptation: the role of osmolytes in bacterial stress and virulence. FEMS Microbiology Reviews, 26(1):49-71, 2002.

51. Erhard Bremer and Reinhard Krämer. Responses of Microorganisms to Osmotic Stress. https://doi.org/10.1146/annurev-micro-020518-115504, 73:313-334, 2019.

52. Luciano F. Huergo and Ray Dixon. The Emergence of 2-Oxoglutarate as a Master Regulator Metabolite. Microbiology and Molecular Biology Reviews, 79(4):419-435, 2015.

53. Wongi Seol and Aaron J. Shatkin. Escherichia coli kgtP encodes an alpha-ketoglutarate transporter. Proceedings of the National Academy of Sciences, 88(9):3802-3806, 1991.

54. Dalai Yan, Peter Lenz, and Terence Hwa. Overcoming fluctuation and leakage problems in the quantification of intracellular 2-oxoglutarate levels in Escherichia coli. Applied and Environmental Microbiology, 77(19):6763-6771, 2011.

55. Kaj Frank Jensen, Gert Dandanell, Bjarne Hove-Jensen, and Martin WillemoËs. Nucleotides, Nucleosides, and Nucleobases. EcoSal Plus, 3(1), 2008.

56. Ramses Gallegos-Monterrosa, Stefanie Kankel, Sebastian Götze, Robert Barnett, Pierre Stallforth, and Ákos T. Kovács. Lysinibacillus fusiformis M5 induces increased complexity in Bacillus subtilis 168 colony biofilms via hypoxanthine. Journal of Bacteriology, 199(22), 2017.

57. Elad Noor, Avi Flamholz, Arren Bar-Even, Dan Davidi, Ron Milo, and Wolfram Liebermeister. The Protein Cost of Metabolic Fluxes: Prediction from Enzymatic Rate Laws and Cost Minimization. PLOS Computational Biology, 12(11):e1005167, 2016.

58. Lieselotte Vermeersch, Gemma Perez-Samper, Bram Cerulus, Abbas Jariani, Brigida GalIone, Karin Voordeckers, Jan Steensels, and Kevin J. Verstrepen. On the duration of the microbial lag phase. Current Genetics, 65(3):721-727, 2019.

59. Breah La Sarre, Alexandra L. McCully, Jay T. Lennon, and James B. McKinlay. Microbial mutualism dynamics governed by dose-dependent toxicity of cross-fed nutrients. The ISME Journal 2017 11:2, 11(2):337-348, 2016.

60. Chen Liao, Sergei Maslov, Tong Wang, and Joao B. Xavier. Modeling microbial crossfeeding at intermediate scale portrays community dynamics and species coexistence. PLOS Computational Biology, 16(8):1-23, 2020.

61. Julijana Ivanisevic, Zheng Jiang Zhu, Lars Plate, Ralf Tautenhahn, Stephen Chen, Peter J. O'Brien, Caroline H. Johnson, Michael A. Marletta, Gary J. Patti, and Gary Siuzdak. Toward 'Omic scale metabolite profiling: A dual separation-mass spectrometry approach for coverage of lipid and central carbon metabolism. Analytical Chemistry, 85(14):6876-6884, 2013.

62. Héctor Gallart-Ayala, loana Konz, Florence Mehl, Tony Teav, Aikaterini Oikonomidi, Gwendoline Peyratout, Vera van der Velpen, Julius Popp, and Julijana Ivanisevic. A global HILICMS approach to measure polar human cerebrospinal fluid metabolome: Exploring genderassociated variation in a cohort of elderly cognitively healthy subjects. Analytica Chimica Acta, 1037:327-337, 2018.

63. Hiroshi Tsugawa, Mitsuhiro Kanazawa, Atsushi Ogiwara, and Masanori Arita. MRMPROBS suite for metabolomics using large-scale MRM assays. Bioinformatics, 30(16):2379-2380, 2014.

64. Warwick B. Dunn, David Broadhurst, Paul Begley, Eva Zelena, Sue Francis-Mcintyre, Nadine Anderson, Marie Brown, Joshau D. Knowles, Antony Halsall, John N. Haselden, Andrew W. Nicholls, Ian D. Wilson, Douglas B. Kell, and Royston Goodacre. Procedures for large-scale metabolic profiling of serum and plasma using gas chromatography and liquid chromatography coupled to mass spectrometry. Nature Protocols 2011 6:7, 6(7):1060$1083,2011$. 